\title{
A fast switch, combiner and narrow-band filter for high- power millimetre wave beams
}

\author{
W. Kasparek ${ }^{1}$, M.I. Petelin ${ }^{2}$, D.Yu Shchegolkov' ${ }^{2}$, V. Erckmann ${ }^{3}$, \\ B. Plaum ${ }^{1}$, A. Bruschi ${ }^{4}$, ECRH groups at IPP Greifswald ${ }^{3}$, FZK \\ Karlsruhe $^{5}$, and IPF Stuttgart ${ }^{1}$. \\ ${ }^{1}$ Institut für Plasmaforschung, Universität Stuttgart, Pfaffenwaldring 31, D-70569 \\ Stuttgart, Germany \\ ${ }^{2}$ Institute of Applied Physics, RAS, 603950 Nizhny Novgorod, Russia \\ ${ }^{3}$ Max-Planck-Institut für Plasmaphysik (IPP), EURATOM-Association, D-17491 \\ Greifswald, Germany \\ ${ }^{4}$ Istituto di Fisica del Plasma, EURATOM-ENEA-CNR Ass., via R. Cozzi 53, 20125 \\ Milano, Italy \\ ${ }^{5}$ Forschungszentrum Karlsruhe, Association EURATOM-FZK, IHM, D-76021 \\ Karlsruhe, Germany \\ e-mail: walter.kasparek@ipf.uni-stuttgart.de
}

\begin{abstract}
A fast directional switch (FADIS) is described, which allows controlled switching of high-power microwaves between two outputs. A possible application could be synchronous stabilization of neoclassical tearing modes (NTM). Generally, the device can be used to share the installed EC power between different types of launchers or different applications (e.g. in ITER, midplane / upper launcher). The switching is performed electronically without moving parts by a small frequency-shift keying of the gyrotron (some tens of $\mathrm{MHz}$ ), and a narrow-band diplexer. The device can be operated as a beam combiner also, which offers attractive transmission perspectives in multi-megawatt ECRH systems. In addition, these diplexers are useful for plasma diagnostic systems employing high-power sources due to their filter characteristics.

The principle and the design of a four-port quasi-optical resonator diplexer is presented. Lowpower measurements of switching contrast, mode purity and efficiency show good agreement with theory. Preliminary frequency modulation characteristics of gyrotrons are shown, and first results from high-power switching experiments using the ECRH system for W7-X are presented.

Key words: Diplexer, Power combiner, Millimeter Wave transmission, Electron cyclotron Heating.
\end{abstract}

PACS numbers: 52.50.Gj， 52.35.-g， 84.40.-x， 42.79.Ci

\section{Introduction}

An attractive feature of electron cyclotron resonance heating (ECRH) and current drive (ECCD) systems for tokamaks is the control of MHD instabilities by directing narrow EC wave beams to the resonant layer [1]. For ITER, the suppression of neo-classical tearing modes (NTM) is a main purpose of ECCD applied from the upper launchers. The highest efficiency for NTM stabilization is reached when ECCD is applied in the center (the "O-point") of the island. As the islands rotate with frequencies of typically 1 to $10 \mathrm{kHz}$, injection of the launched power synchronous with the rotating islands may be required [2].

Up to now, synchronous current drive is performed by power modulation of the gyrotron [1], with the disadvantage that half of the installed power is wasted. An alternative for power modulation could be synchronous toggling of the gyrotron power between two launchers directing the beam to poloidal or toroidal planes, which are about $180^{\circ}$ apart from each other with respect to the phase of the NTM. Alternatively, the power of one output can be used for synchronous NTM stabilization, while the other 
output feeds a launcher for an independent ECRH or ECCD experiment (e.g. ITER, switching between the upper and the midplane launcher).

The switching is performed with a fast directional switch (FADIS), while the source operates continuously. The FADIS is based on a small frequency-shift keying of the gyrotron between $f_{1}$ and $f_{2}$ performed by modulation of the gun anode or the beam acceleration voltage, and a narrow-band frequency diplexer, which directs an input beam into one of two output channels [3], as sketched in figure 1a. Note that for the tiny frequency shifts $f_{1}-f_{2}=\Delta f_{\mathrm{s}}$ of some tens of $\mathrm{MHz}$ needed for the switching, no remarkable change of the deposition radius in the plasma occurs.

As any diplexer can be designed as four-port device, two gyrotrons can be fed into it. If both gyrotrons are shifted between frequencies $f_{1}$ and $f_{2}$, but in opposite phase ("push-pull"), then the power of both gyrotrons is combined into one of the two outputs, and is switched between output 1 and 2 in the rhythm of the frequency-shift keying (see figure 1b). Thus, depending on the experiment, there is no need to increase the number of launchers, else one could even reduce the number of launchers by using this device.

As long-term perspective, switching of a high-power beam generated from several continuously operating sources can be provided by using a multiplexer fed by a set of frequency-controlled gyrotrons $[3,4,5,6,7]$. Infinitely variable beam steering could become possible with a phased array fed by a set of gyrotrons (or high-order-mode gyroklystrons) which are phase locked to a common low-power reference oscillator via electrically controlled phase shifters $[4,5,7,8,9,10]$.

Note that the diplexers discussed here are of interest as well for plasma diagnostics especially when they employ a combination of high-power sources and sensitive receivers. For the measurement of the power deposition zone in NTM stabilization experiments, the "Line of Sight" scheme [11] exploits the gyrotron transmission line in the reverse direction as an electron cyclotron emission (ECE) antenna, thus ensuring that the ECE observed originates from the same location as where the ECRH is deposited. The high-power beam can be fed via the resonant channel (corresponding to $f_{2}$ in figure 1a, but reverse direction) of the diplexer to the launcher, while the ECE signal is coupled out via the nonresonant channel $\left(f_{1}\right.$ in figure 1a) to the detection system. This scheme decouples the source from the receiver, and the notch-filter function of the non-resonant channel suppresses the gyrotron stray radiation in the receiver with high efficiency, in contrast to quartz plates used for this purpose [12]. Collective Thomson scattering systems [13] may additionally benefit from this device, as the resonant channel can be used to purify the frequency spectrum of the gyrotron source.

High-power diplexers can be realized in various forms $[4,14,15]$. In this paper, a FADIS prototype based on a compact quasi-optical cavity is discussed. Calculations and low-power measurements are presented. Results of preliminary frequency modulation experiments are shown, and first high-power tests using the ECRH system for W7-X are described. A note on the integration of such a FADIS into (corrugated waveguide) transmission lines follows. Other concepts for realizing a resonant diplexer are specified in section 7. Finally, future development steps are discussed.

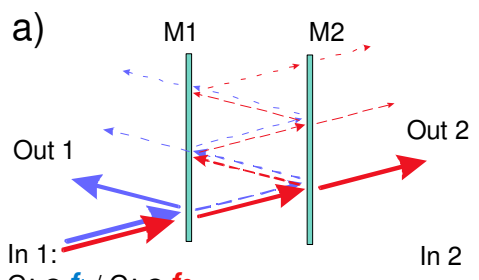

GA@f1/GA@f2

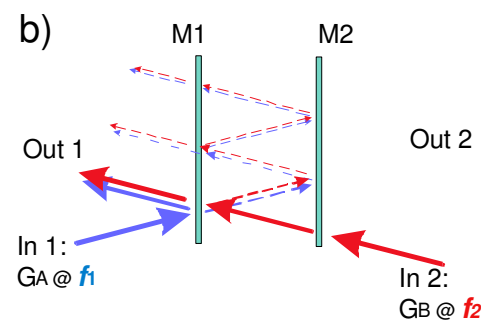

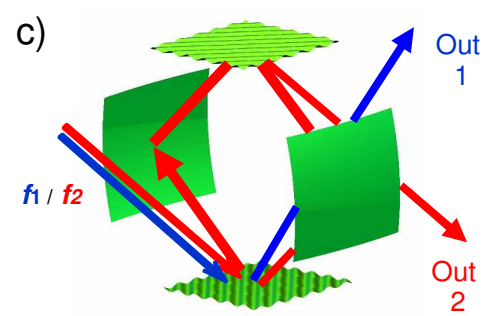

Figure 1: FADIS based on the Fabry-Perot interferometer. In the principle sketches a and b, M1 and M2 are partially reflecting mirrors, In 1,2 and Out 1,2 are (directions of) input and output beam, and $f_{1}$ and $f_{2}$ are non-resonant and resonant frequencies of the beams, respectively. a) Principle for switching by frequency-shift keying, b) combination of two sources with small frequency difference, c) high-power design using a 4-mirror quasi-optical cavity with grating couplers. 


\section{Design of the Quasi-optical FADIS}

The principle design for a resonant diplexer/FADIS is sketched in figure 1. For high power applications, where only reflecting optics are available, we use a version consisting of a quasi-optical ring resonator with a high Q-factor with two integrated diffraction gratings as input and output couplers (figure 1c). If we denote the scattering coefficients of the gratings for $0^{\text {th }}$ order as $r_{0}$, for $-1^{\text {st }}$ order as $r_{1}$, and take internal (ohmic, diffraction) losses into account by the scattering coefficient $r_{\mathrm{q}}$ for the unloaded resonator, then the quality factor $Q$ of the resonator is given by

$$
Q=-\frac{k \cdot L}{2 \cdot \ln \left(\left|r_{0}^{2}\right|\right)+2 \cdot \ln \left(\left|r_{q}\right|\right)}
$$

Here, $L$ is the round-trip length of the resonator and $k$ is the wave-vector of the radiation. The amplitude transmission coefficients from the input to output 1 and 2, respectively, are given by

$$
t_{1}(\Delta k)=r_{0} \cdot \frac{1-\left|r_{q}\right| \exp (i \Delta k L)}{1-\left|r_{q} r_{0}^{2}\right| \exp (i \Delta k L)}, \quad t_{2}(\Delta k)=\frac{r_{1}^{2} \sqrt{r_{q}} \exp (i \Delta k L / 2)}{1-\left|r_{q} r_{0}^{2}\right| \exp (i \Delta k L)}
$$

with $\left|r_{1}^{2}\right|+\left|r_{0}^{2}\right|=1$, and $\Delta k=2 \pi \cdot \Delta f / c, \Delta f$ being the detuning of the resonator from its resonant frequency. Note that all angles of incidence and diffraction on the grating are equal $\left(45^{\circ}\right.$ in the present design). For high-power applications, the normalized power in the cavity is of interest, which in resonance condition is given by:

$$
\frac{P_{\text {Re } s 1, \max }}{P_{I n}}=\left|\frac{t_{2}(0)}{r_{1}}\right|^{2}
$$

in the region before the beam comes to the grating working for resonance output and

$$
\frac{P_{\mathrm{Re} s 2 \max }}{P_{I n}}=\left|\frac{t_{2}(0)}{r_{1}} \cdot r_{0}\right|^{2}
$$

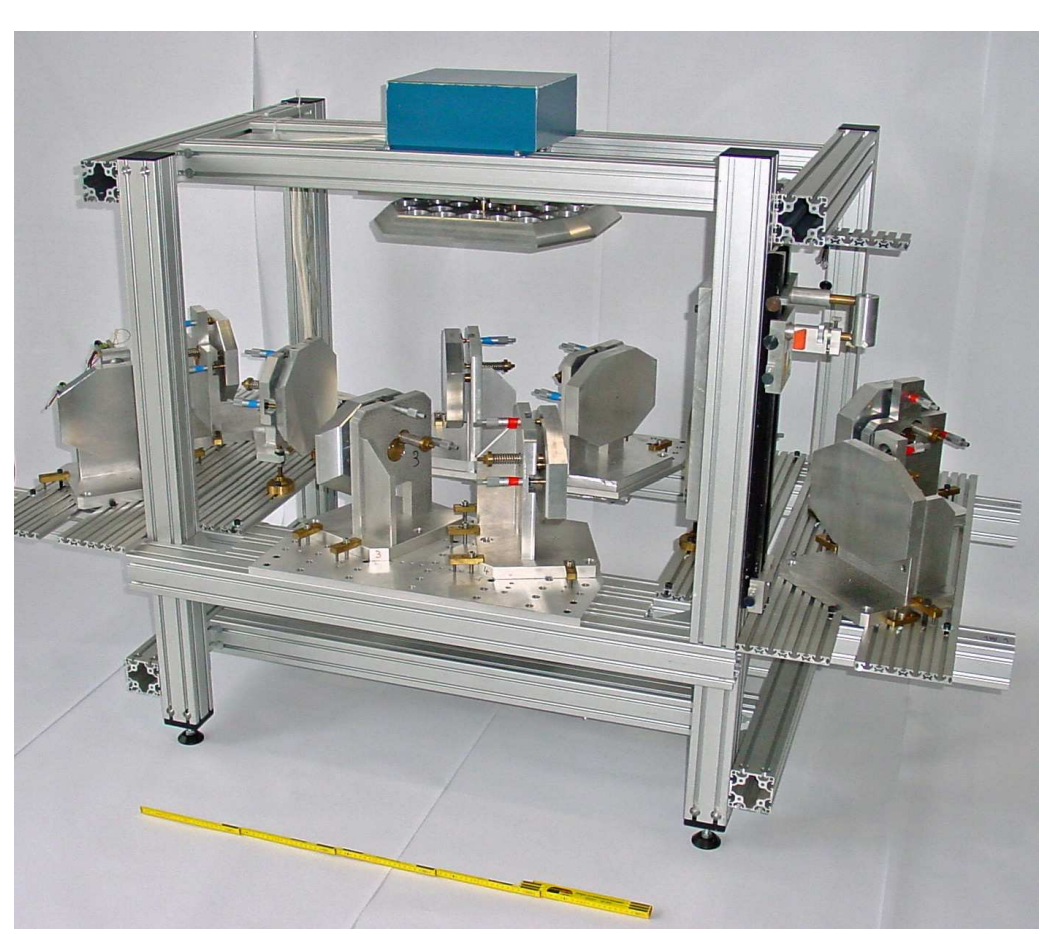

Figure 2: Set up of the quasi-optical diplexer consisting of the ring resonator (top and - non-visible - bottom mirror and left and - nonvisible - right grating mirror, as well as 4 two-mirror matching optics for incoming and outgoing beams. after being reflected from this grating.

This type of diplexer has been investigated in detail for highpower multi-channel transmission $[5,6]$. As prototype for a high-power switch, a diplexer/combiner for $140 \mathrm{GHz}, 1 \mathrm{MW}$ was designed, consisting of a ring resonator with $L=2.4 \mathrm{~m}$, and two coupling gratings with (designed) efficiencies in $0^{\text {th }}$ and $-1^{\text {st }}$ order of $R_{0}=\left|r_{0}\right|^{2}=$ 0.755 and $R_{1}=\left|r_{1}\right|^{2}=0.245$, respectively. This results in a free spectral range (distance of resonances) of $125 \mathrm{MHz}$. The beam waist radii on the grating mirrors in the resonator are of $\mathrm{w}_{0}=32.0 \mathrm{~mm}$ and $\mathrm{w}_{0}=45.3$ $\mathrm{mm}$, respectively. At $1 \mathrm{MW}$ input power, the maximum electric field in the resonator (as 
calculated from (3) and including the fields of the coupled beams) corresponds to an energy-flux density of $0.83 \mathrm{MW} / \mathrm{cm}^{2}$, which is below the $1 \mathrm{MW} / \mathrm{cm}^{2}$ threshold of atmospheric air breakdown. This allows the high-power test of the device under normal atmosphere. Therefore, the matching mirrors for the incident beams from two gyrotrons and the two output beams were designed to fit into the ECRH installation for W7-X at IPP Greifswald [16]. The complete set up is shown in figure 2.

\section{Low-Power Test}

Detailed low-power measurements using a vector network analyzer and calorimetry have been performed. The input beam was coupled by a scalar horn and a matched mirror. A good agreement with the calculations is achieved. As an example, figure 3 shows a calorimetric measurement of the transmission functions for the resonant and non-resonant channel, yielding efficiencies of about $93 \%$ and $98 \%$, respectively. From the fits of theoretical curves (2) to the measurements, the following results were obtained: The grating depth was slightly lower due to a low number of data points for manufacturing, the corresponding efficiency in minus first order was $R_{1}=0.218$. The round-trip loss in the resonator was measured to be $<1.3 \%$ and is near to the theoretical value of about $1 \%$ determined by the ohmic loss, atmospheric attenuation, and beam truncation of the resonator mirrors. The measured quality factor (eq. 1 ) is $Q \approx 14000$; thus, for the FADIS application, a good switching contrast can be reached for $\Delta f_{\mathrm{s}}>30 \mathrm{MHz}$.

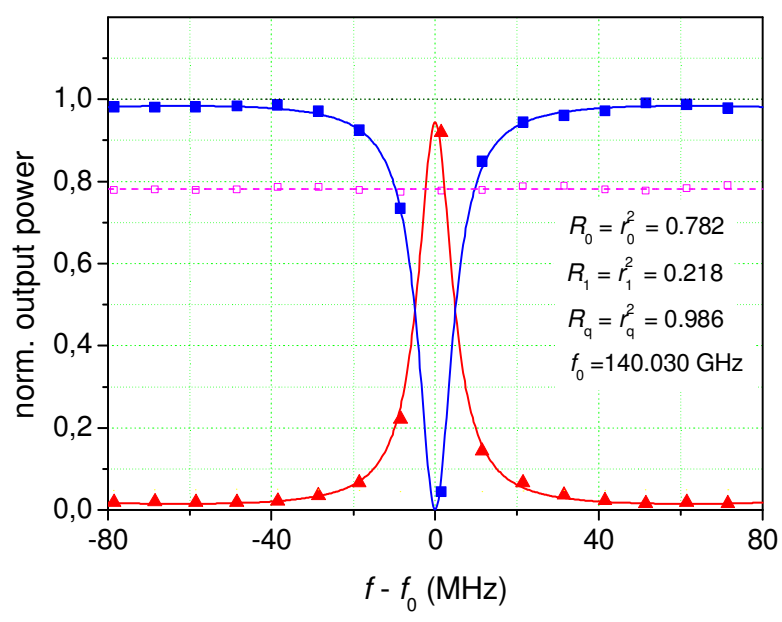

Figure 3: Low-power measurement of the transmission functions of the diplexer. Squares: non-resonant output 1; triangles: resonant output 2; small open squares: $0^{\text {th }}$-order efficiency of the coupling grating. Lines: Calculated curves (eq. 2) with parameters given in the plot.

A broadband frequency scan of the transmission function is plotted in figure 4 . The envelope of the transmission maxima shows that the operating range (97-\%-bandwidth) of the device is at least 500 $\mathrm{MHz}$, i.e. fully sufficient for the frequency variation of a monomode gyrotron ( $c f$. chapter 4.). Note that the reduction of transmission at frequencies outside the centre frequency is caused by the dispersion of the coupling gratings, leading to a frequency-dependent misalignment for larger

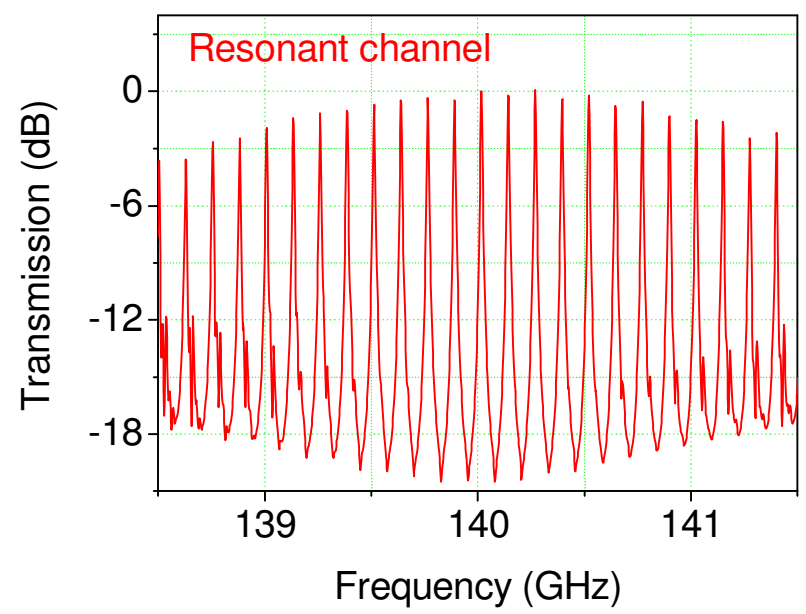

Figure 4: Broadband frequency scan of the transmission function for the resonant channel showing a bandwidth of more than $500 \mathrm{MHz}$. frequency offsets.

At the outputs of the FADIS, beam patterns were recorded with a small waveguide probe and an $\mathrm{x}$-y-scanner. The patterns measured at the maxima of the non-resonant and the resonant outputs (see figure 5) show a high quality of the output beams. The Gaussian beam mode analysis yields a $\mathrm{TEM}_{00}$ content of $99.0 \%$ and $99.8 \%$, respectively.

The peak amplitudes of the patterns measured at the minima of the non-resonant and the resonant outputs have levels, which are $15 \mathrm{~dB}$ and $20 \mathrm{~dB}$ lower than the corresponding values for maximum output, and thus confirm the contrast between minimum and maximum transmission measured by calorimetry (figure 3 ). 


\section{Gyrotron Frequency Control}
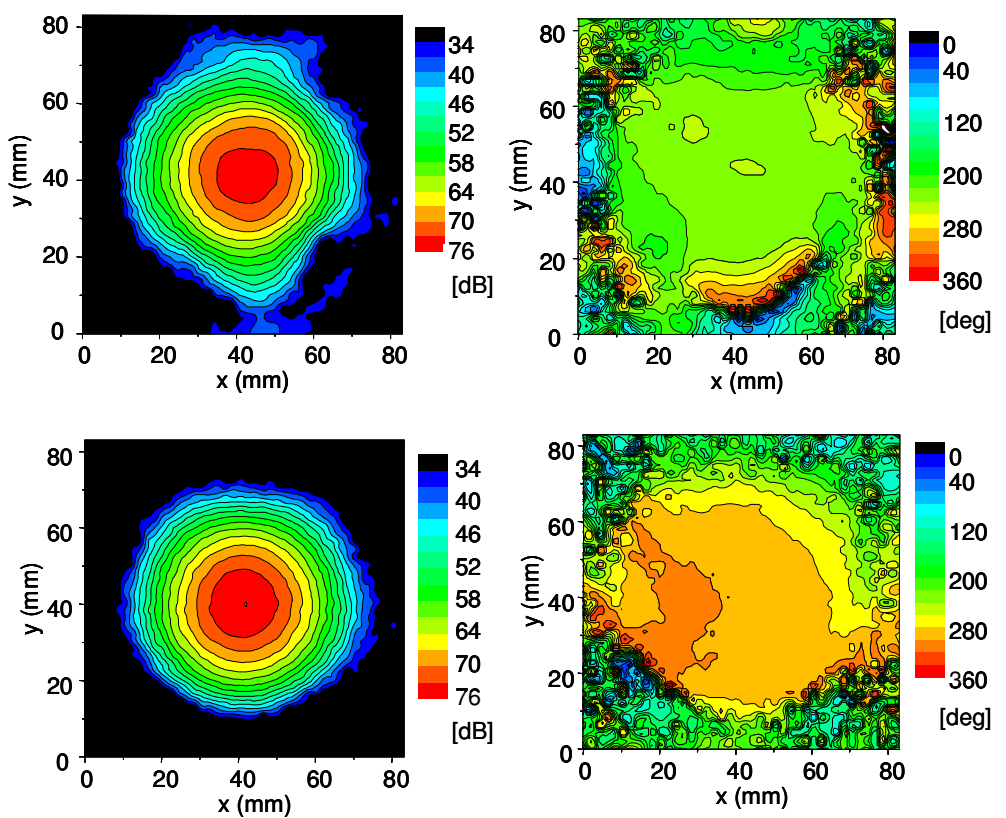

Figure 5: Amplitude (left) and phase (right) patterns of the output beams of the non-resonant channel (top) and the resonant channel (bottom), measured at the frequencies for maximum transmission.
A fast frequency control of a free running single-mode gyrotron can be achieved only by changing an operation voltage: the change transforms electron beam parameters, including the reactive part of $\mathrm{RF}$ conductivity, and finally results in a frequency shift [8]. For the high-power test of the FADIS, the TED prototype gyrotron "Maquette" [17] with depressed collector was used; the control voltage is applied between the collector and the RF cavity ("body") [18].

Measurements of the frequency characteristics of the TED Maquette gyrotron show the following: For about $0.5 \mathrm{~s}$ after switch-on, the gyrotrons exhibit a (output power-dependent)

strong frequency chirp of up to $300 \mathrm{MHz}$ (figure 6a), which is due to heating and thermal expansion of the cavity. After thermalization of the cavity ( $>1 \mathrm{~s})$, the frequency is stable within $5 \mathrm{MHz}$ for the investigated pulse lengths of up to $160 \mathrm{~s}$. If a modulation is applied by the body voltage modulator [18], the frequency variation is, obviously, accompanied by a modulation of the output RF power. The frequency swing depends not only on the voltage swing but also on the operational conditions, especially the actual frequency. The frequency during the switching does not exactly follow the
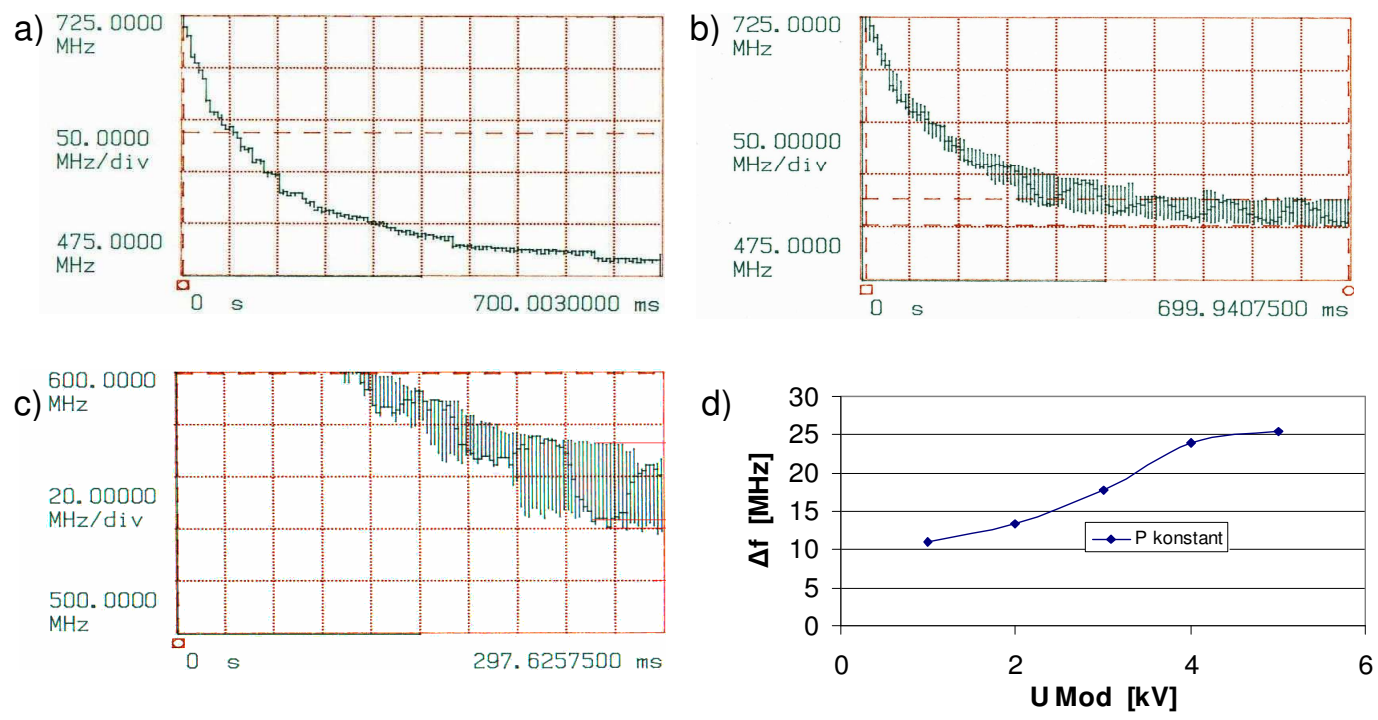

Figure 6: Temporal variation of the frequency of the TED prototype gyrotron "Maquette" at $U_{\text {acc }}=81.5 \mathrm{kV}$. Note that the real gyrotron frequency is obtained by adding the local oscillator frequency of $139.65 \mathrm{GHz}$. a) no modulation; b) and c) with modulation $\left(\Delta U_{\mathrm{B}}=4 \mathrm{kV}, f_{\mathrm{MOD}}=5 \mathrm{kHz}\right.$ square wave; d) frequency shift as function of the voltage modulation swing. 
voltage variation, but rather jumps abruptly from one frequency level to the other; in some cases, the switching occurs over several frequency steps. A reason could be that the frequency of a gyrotron is influenced by reflections back to the cavity, as well as the phase of the reflected waves [19]. An

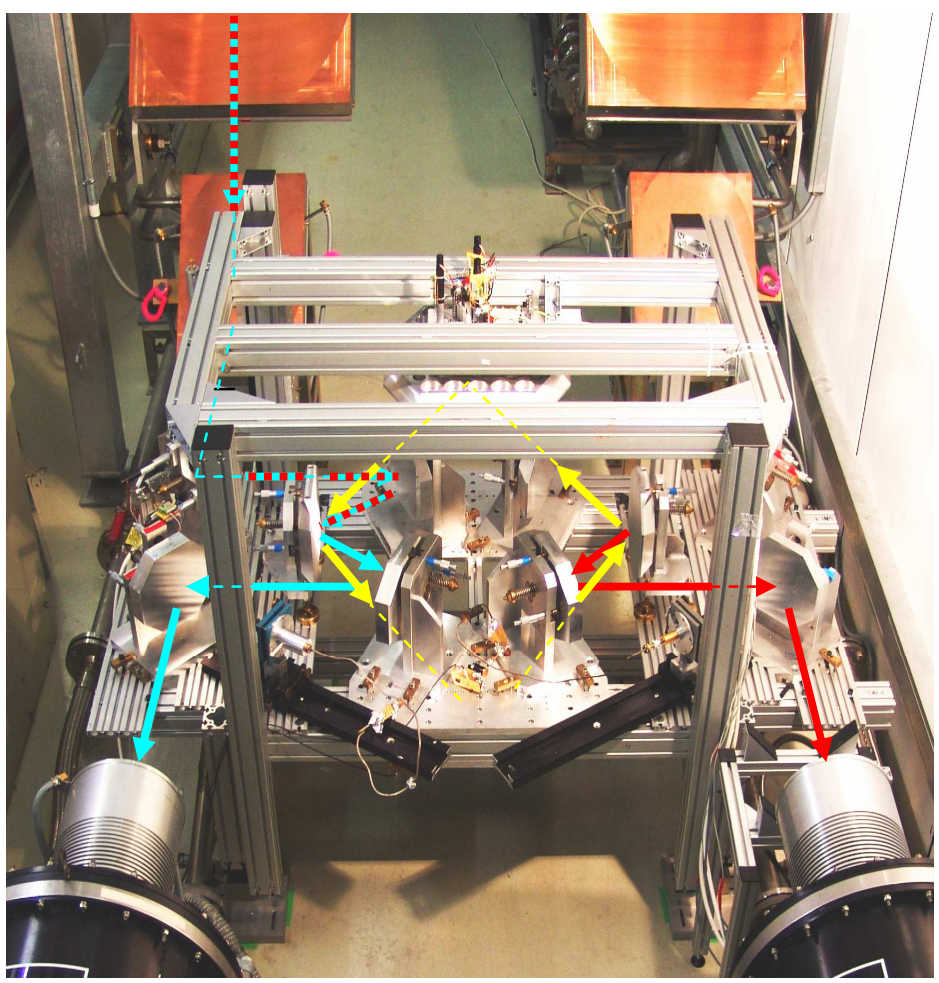

Figure 7: Photograph of the FADIS installed in the beam duct of the ECRH system on W7-X. The input beam is coupled from the rear in to the resonator, the output beams are focussed to absorbing loads in the foreground left (output 1) and right (output 2). example for a frequency measurement with a voltage modulation of $4 \mathrm{kV}, 5$ $\mathrm{kHz}$ square wave resulting in a frequency-shift keying of $\Delta f_{\mathrm{s}} \approx 30$ $\mathrm{MHz}$ is shown in figures $6 \mathrm{~b}$ and $6 \mathrm{c}$. The typical frequency shift as function of the body voltage swing is displayed in figure $6 \mathrm{~d}$.

\section{High-Power Test of the FADIS}

For the high-power test, the FADIS was equipped with a motorized drive of the upper resonator mirror which allowed the tuning of the transmission curve with respect to the gyrotron frequency. The device was installed in front of the cw calorimetric loads in the underground transmission duct of the ECRH system for W7-X, as shown in figure 7. The incident beam was coupled with two matching mirrors to the FADIS, and the output beams were dumped into the loads. Owing to the limitation of the pulse length (typically $0.7 \mathrm{sec}$ ) because of the use of uncooled $\mathrm{Al}$ mirrors also in the resonator, grating couplers on the output mirrors served as power monitors.

Most of the experiments were carried out in a power range of $300 \mathrm{~kW}$ to $600 \mathrm{~kW}$. Here, the device operated without remarkable problems. Arcing occured very infrequently, although the maximum power density in the beam near to the grating surface was about $0.5 \mathrm{MW} / \mathrm{cm}^{2}$.

For a confirmation of the transmission functions, the chirp of the gyrotron was used to sweep the frequency over the resonance. The result is shown in figure 8; a qualitative agreement with the expectations is obtained. Note that the frequency variation of the gyrotron is not continuous (cf. figure 6a), which explains the jumps in the measurement.
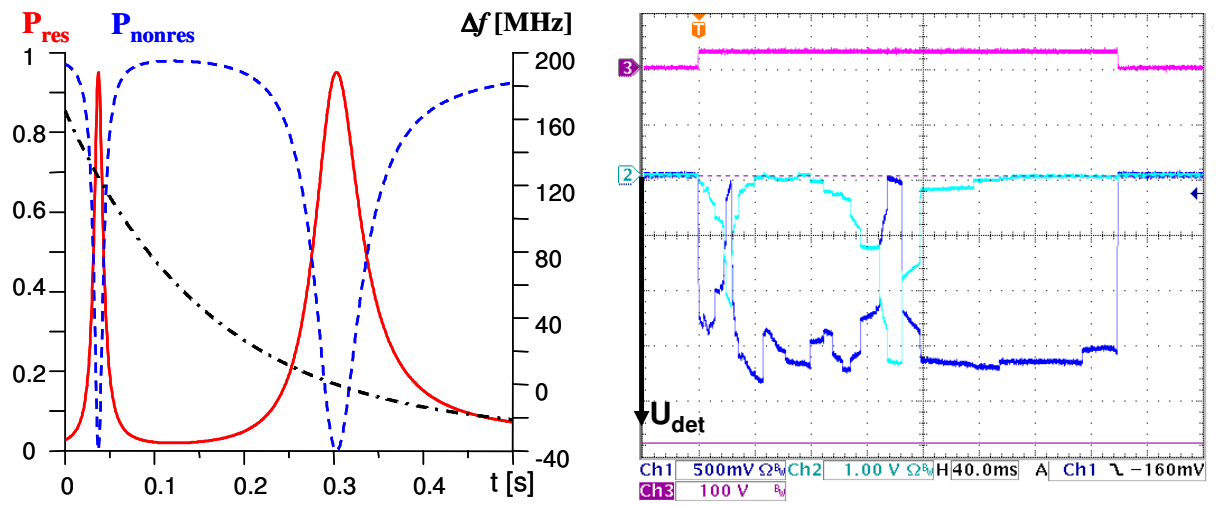

Figure 8: Temporal variation of the FADIS outputs due to the frequency chirp of the gyrotron after switch-on ( $c f$. figure 6a), showing qualitatively the transmission functions for output 1 (dark blue) and 2 (light blue) under highpower conditions. 


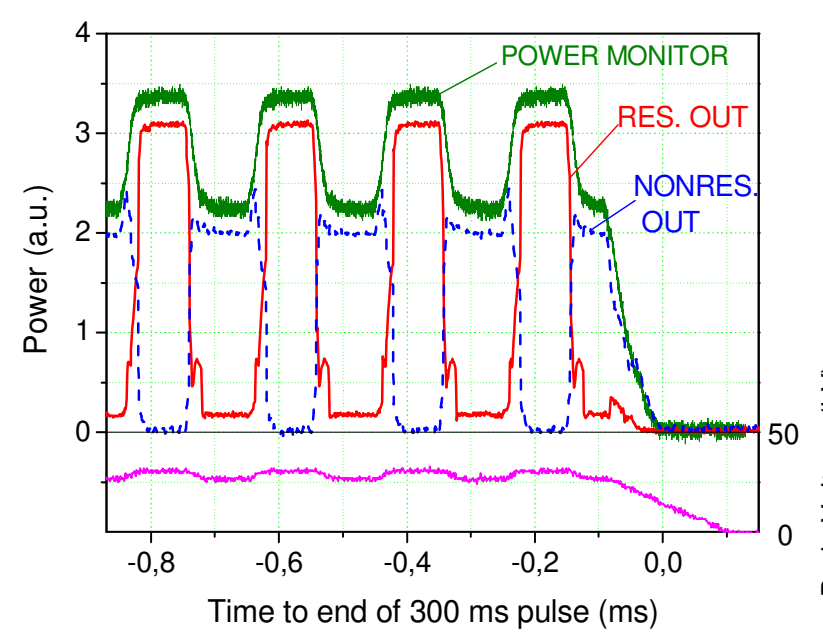

Figure 9: Power signals from output 1 (blue, dashed) and out put 2 (red, solid), shown at the end of a $300 \mathrm{~ms}$ pulse with $U_{\text {acc }}=81.5 \mathrm{kV}, \Delta U_{\mathrm{B}}=4 \mathrm{kV}, f_{\mathrm{MOD}}=5 \mathrm{kHz}$ square wave. The enveloping trace (olive) is the signal from the gyrotron power monitor. The lower trace shows the body voltage.
To measure the switching performance, the resonator was tuned such that the gyrotron frequency was near to a resonant frequency at the end of the pulse. Body voltage modulation in the range of $1 \mathrm{kV} \leq \Delta U_{\mathrm{B}} \leq 5$ $\mathrm{kV}$ square wave with frequencies of $1 \mathrm{kHz} \leq$ $f_{\text {MOD }} \leq 20 \mathrm{kHz}$ was applied. An example $\left(\Delta U_{\mathrm{B}}=4 \mathrm{kV}, f_{\mathrm{MOD}}=5 \mathrm{kHz}\right)$ is shown in figure 9, where the power signals for the outputs and the input are plotted. A high switching contrast of $94 \%$ in the resonant and $99 \%$ in the non-resonant output is measured. Even at $20 \mathrm{kHz}$, switching could be demonstrated, however with less contrast, as the slew rate of the body-voltage modulator (usually $600 \mathrm{~V} / \mu \mathrm{s}$ ) had to be reduced for reasons of electromagnetic compatibility in the main HV power supply system.

\section{Integration of resonant diplexers into transmission lines}

Especially for the application in larger ECRH systems, it is necessary to develop compact diplexers and integrate them into the transmission lines without interference with neighbouring lines.

Quasi-optical versions of the FADIS would be ideal solutions for optical transmission systems, as they can be designed as an integral part of the whole system. For transmission systems using circular corrugated waveguides, the matching of quasi-optical diplexers can be done with appropriate optics, however, such arrangements may become bulky. An elegant and very compact possibility is the synthesis of waveguide mode converters fed by a circular $\mathrm{HE}_{11}$ mode, which produce a field pattern which perfectly matches the $\mathrm{TEM}_{00}$ input field of the diplexer $[20,21,22]$. This type of coupling
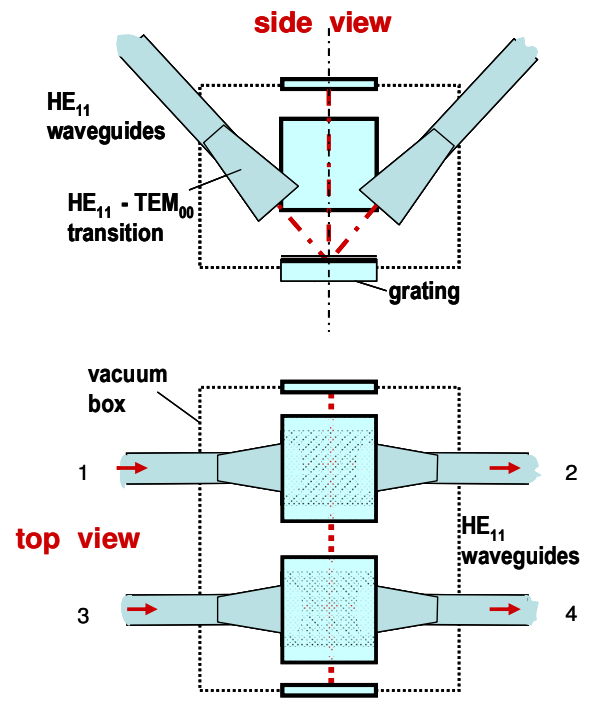

Figure 10: Compact version of a quasi-optical FADIS employing $\mathrm{HE}_{11}$ waveguide mode converters to project a $\mathrm{TEM}_{00}$-mode with plane phase front onto the coupling gratings. The mirrors are screwed into a precisely machined vacuum box to avoid alignment tools. A movement of one mirror normal to its surface by about one wavelength allows the tuning according to the gyrotron frequency. would allow very compact matching e.g. to the resonant diplexer. An example for the design of a diplexer/combiner with two inputs and outputs by corrugated circular waveguides is sketched in figure 10 . If the resonator mirrors are integrated into the walls of the vacuum chamber needed for evacuated transmission lines, a very compact FADIS design is obtained. Depending on the situation, ports 1 and 3 can be used as inputs for the gyrotrons and ports 2 and 4 as outputs. If ports 1 and 4 are used as inputs, and ports 2 and 3 as outputs, the diplexer can be designed to work 
for arbitrary polarization, as the coupling gratings can be tailored to have identical efficiency in TE and TM polarization (perpendicular and parallel to the grooves, respectively), and the phase shift between polarization components on the gratings is compensated by their orientation in the resonator. Alternatively, one of the resonator mirrors can be corrugated to compensate for the phase shift on the gratings.

It should be noted, however, that the two outputs of the FADIS often need different polarization, as either the antennas or the applications corresponding to the outputs are different. Therefore, in general, the fast switch has to be installed in the transmission system between the sources and the elements for polarization control. Thus, designs for linear polarization can be used as well.

\section{Other versions of resonant diplexers-combiner}

The resonant diplexer discussed in this paper may be realized in different ways, mainly depending on the power splitting device used in the resonator.

One possible splitter available is the square or rectangular corrugated waveguide of length $L=2 D^{2} / \lambda$, where $\mathrm{D}$ is the waveguide aperture and $\lambda$ the wavelength. Its length is half that of a remote steering antenna, that has been already studied and tested at high power [23, 24]. When one beam is launched at the input with angular (with angle less than a few degrees) or side input (figure 11a), two beams of equal (half) power emerge from the waveguide output. This is an effect of the more general imaging/splitting property of overmoded waveguides for paraxial propagation [25, 26]. The waveguide section can be considered a four-port device, with a second input found symmetrically with respect to the first [27].

A device equivalent to the quasi-optical FADIS, described in section 2, can be realized with a pair of square corrugated waveguide splitters and mirrors (figure 11b; device shown in solid lines) [28]. The device, which for power handling reasons must have a length of a few meters, has a resonant loop with length of around $2 L$. Given the power splitting ratio of one half for the single splitter, a non-sufficient power switching contrast of $8: 1$ for the two channels at non-resonant frequencies is obtained (figure 12a). The addition of a third splitter to form a second loop in cascade (figure $11 \mathrm{~b}$, considering additional mirrors and waveguide shown in dashed lines) helps, when tuned to the first loop, in achieving a sufficient contrast (ideally 98:2 in non-resonant frequencies, higher at resonance, see figure $12 \mathrm{~b}$ ). This setup has the advantage to have inputs at one side and outputs on the other side of the waveguide bundle, helping integration into transmission lines, at which the waveguide can be connected either directly (side input) or by a suitable optics (for angular input). The angular input is

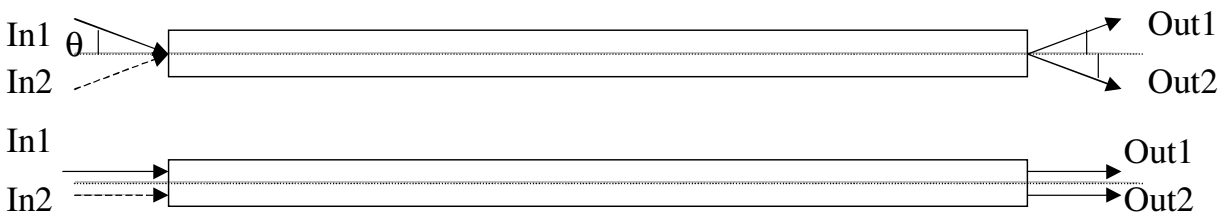

a)

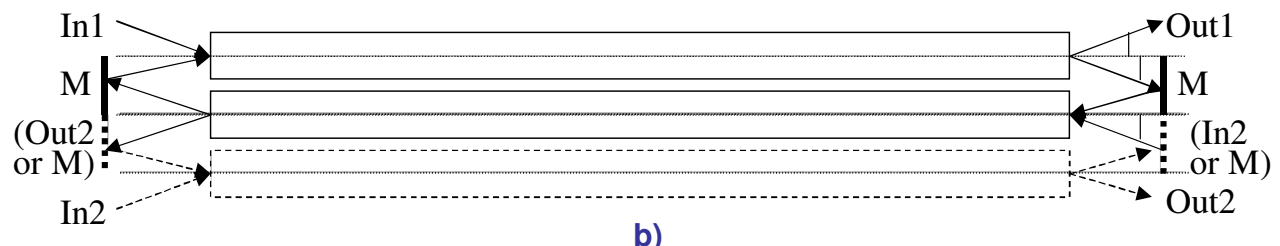

b)

Figure 11: Use of the rectangular corrugated waveguide as 3-dB beam splitter (a), and resonating combiner setup with two (solid lines) and three waveguide sections (b).

usually preferred in order to limit the waveguide length, at equal input beam size, since for side input $D$ is doubled and $L$ results four times longer. The transmission function for two tuned loops has a characteristic form (figure 12b) that presents two maxima, and a relative minimum at half power in between. 


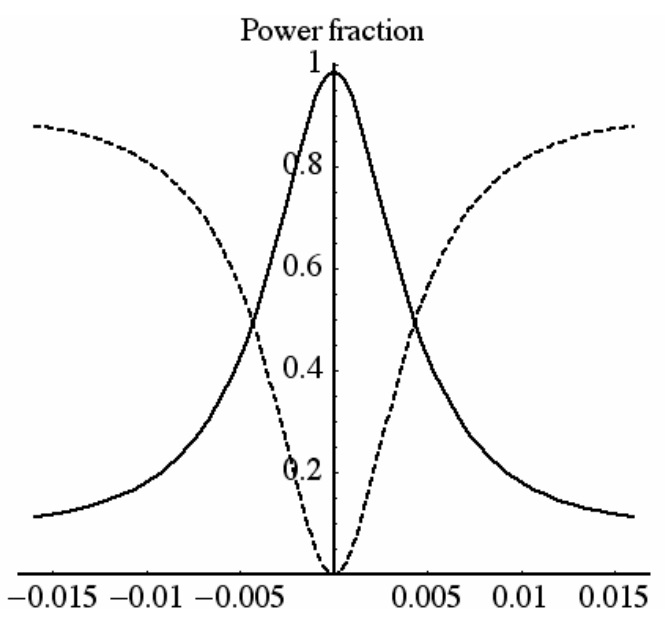

(a)

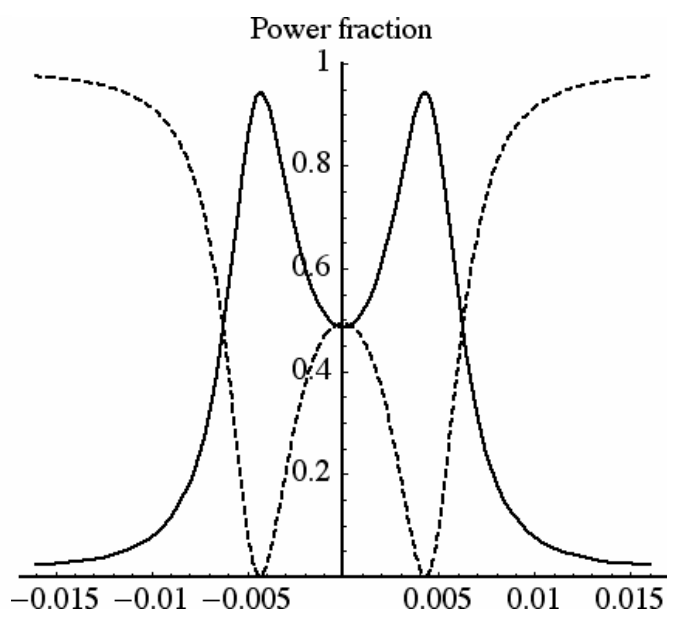

(b)

Figure 12: Power fractions at Out1 (dashed line) and Out2 (solid line) for (a) the two- and (b) the three-waveguide setup, considering losses of around $1.5 \%$ per loop.

The two maxima allow more flexibility when putting diplexers in cascade [29], while the relative minimum can be exploited to share the power equally between two channels in a relatively stable region of frequencies. This possibility may prove useful for even power distribution between transmission lines, thus reducing stresses in transmission line components in case not all the sources are used, or to realize complete interference in multi-beam components or antennas $[4,7]$. The twoloop arrangement can be equally built for the FADIS device described in section 2, adding a grating coupler to form another resonant loop.

One advantage of the waveguide setup is the long path of the resonating loop that is achieved with a relatively compact width of the device: this allows smaller frequency variations, thus low loss due to HV modulation, which compensates the higher insertion loss expected.

Another type of splitter is the dielectric sheet, which for high-power and long pulses or CW is typically a CVD diamond sheet, usually exploited in resonant windows with edge cooling [30]. Even if very thin (less costly) sheets should be required, their use can be complicated by their thinness and the dependence of the splitting factor on polarization. They could allow relatively simple arrangements like the one shown in figure 13.

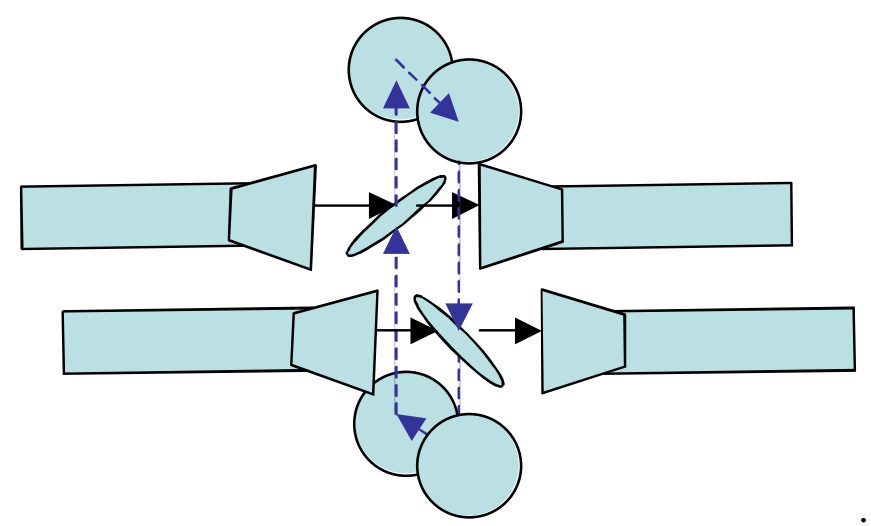

Figure 13: Resonating diplexer/combiner realized with dielectric plate beam splitters: the resonating loop is shown with dashed arrows. 


\section{Conclusion}

Fast switching of the combined beam from one output channel to another seems attractive for adaptive suppression of NTM modes. Switching of the heating power between two launchers increases the flexibility of any ECRH system. Combining of outputs of sub-systems of gyrotrons into one transmission line is of general interest and has many attractive applications. Resonant diplexers in plasma diagnostics employing gyrotrons may enhance the system performance.

The first tests of the quasi-optical FADIS show good agreement with theory and confirm the possibility of fast switching of high-power millimetre wave beams. An experiment for combination of two gyrotrons is in preparation. The results motivate the development of fast switches until maturity, including the following tasks: (i) Investigation of various diplexer concepts with respect to switching contrast and integration into high-power waveguide and optical transmission lines, without interference with adjacent lines; development of $\mathrm{cw}$ devices [4]; (ii) optimization of the frequency control of gyrotrons with minimal power loss using parameter optimization, induced step-like frequency tuning by tiny reflections and possibly phase locking of the gyrotron [8, 19]; (iii) investigations on the composition of diplexers into a multiplexer-scanner which is capable to combine the output powers of several gyrotrons [9]; (iiii) application of a FADIS at a fusion device; experiments at the tokamaks ASDEX Upgrade in Garching and FTU in Frascati are under discussion.

\section{Acknowledgement}

This work is carried out in the frame of the virtual institute "Advanced ECRH for ITER", which is supported by the Helmholtz-Gemeinschaft deutscher Forschungszentren. The ECRH system for W7-X used for this work is managed by the project PMW, ECRH for W7-X hosted at FZK Karlsruhe (collaboration between FZK Karlsruhe, IPP Garching and Greifswald, and IPF Stuttgart).

\section{References}

[1] Zohm H, Gantenbein G, Giruzzi G, Günter S, Leuterer F, Maraschek M, Meskat J P, Peeters A G, Suttrop W, Wagner D, Zabiego M, and ASDEX-Upgrade Team, ECRH Group, 1999, Experiments on neoclassical tearing mode stabilization by ECCD in ASDEX Upgrade. Nucl. Fusion $39577-580$

[2] La Haye R J, Prater R, Buttery R J, Hayashi N, Isayama A, Maraschek M E, Urso L, and Zohm H, 2006, Cross-machine benchmarking for ITER of neoclassical tearing mode stabilization by electron cyclotron current drive. Nucl. Fusion 46 451-461

[3] Petelin M I and Kasparek W, 2005, Electrically controlled scanning of wave beam produced by gyrotron: Option for plasma fusion experiment. Proc. 6th Int. Vacuum Electronics Conference (IVEC 2005), Noordwijk, The Netherlands, p. 131

[4] Kasparek W, Petelin M I, Erckmann V, Shchegol'kov D Yu, Bruschi A, Cirant S, Thumm M, Plaum B, Grünert M, Malthaner M, 2007, Fast switching and power combination of high-power electron cyclotron wave beams: principles, numerical results and experiments. Fusion Sci. Technol. 52, and references therein

[5] Petelin M.I., G. Caryotakis, A.A. Tolkachev et al, Quasi-Optical Components for MMW Fed Radars and Particle Accelerators, in High Energy Density Microwaves, edited by R.M. Phillips, 1998 AIP Conference Proc. 474304 - 315

[6] Koshurinov Yu I, Pavelyev V G, Petelin M I, Turchin I V, Shchegol kov D Yu, 2005, Diplexer based on open cavity with corrugated mirrors. Tech. Phys. Lett. 31709 - 711

[7] Petelin M, Bruschi A, Erckmann V, Kasparek W, 2006, FADIS: Project for Fast Directional Switching of Discrete High Power Millimeter Wave Beams, Proc. Joint 31st International Conference on Infrared and Millimeter Waves and 14th International Conference on Terahertz Electronics, September 18th to 22nd 2006, Shanghai, China. http://www.sitp.ac.cn/irmmw-thz2006

[8] Golubiatnikov G Yu et al., 2006, Gyrotron frequency control by a phase lock system. Technical Physics Letters 32 650-652

[9] Petelin M I, 2003, Quasi-optics in High-Power Millimeter-Wave Systems. 6th Workshop on High Energy Density and High Power RF, WV, USA, AIP Conference Proc. 691, 251-262 
[10] Ilyakov E, et al., Gyroklystron operating at a sequence of high-order modes, in Strong microwaves in Plasmas, N. Novgorod, ed. by A.Litvak, 2006, Vol. 1, 58-61.

[11] Westerhof E et al., 2005, A generic method for controlled ECRH/ECCD localisation, Proceedings of the $13^{\text {th }}$ Joint Workshop on Electron Cyclotron Emission and Electron Cyclotron Heating, 17-20 May 2004, Nizhny Novgorod, Russia, Ed. A. Litvak, Institute of Applied Physics RAS (2005) 357

[12] Oosterbeek J W et al., 2006, Design of a dedicated ECE diagnostic for feedback control of instabilities by ECRH. In 14th Joint Workshop on Electron Cyclotron Emission and Electron Cyclotron Resonance Heating (EC-14), page 76, Santorini, GR, 2006. Book of Abstracts. http://www.hellasfusion.gr/englishver/ec14 conference.php.

[13] Suvorov E V, Erckmann V, Holzhauer E, Kasparek W, Dryagin Y A, Fil'chenkov S E, Fraiman A A, Geist T, Kick M, Kukin L M, Kostrov A V, Lubyako L V, Shtanyuk A M, Skalyga N K, Smolyakova O B, W7-AS Team, and NBI Team, 1995, "Measurement of the thermal ion feature with collective scattering of $140 \mathrm{GHz}$ gyrotron radiation in the W7-AS stellarator“, Plasma Phys. Control. Fusion, 37 1207

[14] Lesurf J C G., Millimeter wave optics, devices and systems. Adam Hilger, Bristol, 1990 ISBN 085274-129-4, p. 136

[15] Smits F M A, 1993, Power combiners for incoherent waves. Proc. of 8th Joint workshop on ECE and ECRH, Report IPP III/186, Vol. 2, 607 - 621, 1993

[16] Erckmann V, et al., 2007, Electron Cyclotron Heating for W7-X: Physics and Technology. Fusion Sci. Technol., 52291.

[17] Thumm M et al., 2007, EU Megawatt-class 140-GHz CW gyrotron. IEEE Trans. Plasma Sci. 35143 - 153

[18] Brand P and Müller G A, 2003, Circuit design and simulation of a HV-supply controlling the power of $140 \mathrm{GHz} 1 \mathrm{MW}$ gyrotrons for ECRH on W7-X. Fusion Eng. Design 66-68 573 - 577

[19] Antonsen T M, Cai S Y, Nusinovich G S, 1992, Effect of window reflection on gyrotron operation. Phys. Fluids B 44131 - 4139

[20] Shapiro M A and Vlasov S N, 1992, Study of combined transmission line for high power wave generated by a gyrotron in the millimetre wavelength range. Int. J. Electronics $\mathbf{7 2}$ 1127-1133

[21] Graubner T, Kasparek W, Kumric H, 1994, Proc. of the $19^{\text {th }}$ Int. Conf. on Infrared and Millimeter Waves, K. Sakai and T. Yoneyama, editors, JSAP Catalog No. AP 941228, 238 - 239

[22] Sobolev D I, Chirkov A V, Denisov G G, Lukovnikov D A, and Malygin V I, 2005, Minimization of diffraction losses in big gaps of multi-mode waveguides. Int. J. Infrared and Millimeter Waves 26953 965

[23] Chirkov A V, Denisov G G, Kasparek W, and Wagner D, 1998, Simulation and experimental study of a remote wave beam steering system. Proc. 23rd Int. Conf. on Infrared and Millimeter Waves (Colchester) ed T J Parker and S R P Smith, ISBN 0-9533839, 250 - 251

[24] Plaum B, Gantenbein G, Kasparek W, Schwörer K, et al., 2005, High-power tests of a remote steering launcher mock-up at $140 \mathrm{GHz}$. Journal of Physics: Conference Series 25 120-129

[25] Ulrich R and Ankele G, 1975, Self imaging in homogeneous planar optical waveguides, Appl. Phys. Lett. 27337

[26] Bachmann M, Besse PA, Melchior, H, 1994, General self-imaging properties in NxN multimode interference couplers including phase relations. Applied Optics 333905 - 3911

[27] Kuzikov S V, 1998, Wavebeam Multiplication Phenomena to RF Power Distribution Systems of HighEnergy Linear Accelerators. Int. J. Infrared and Millimeter Waves 19 1523-1539

[28] Bruschi A, Cirant S, Moro A, Simonetto A, 2006, High-Power Millimetre-Wave Components Combining Quasi-Optics With Oversized Rectangular Corrugated Waveguide. 14th Joint Workshop on Electron Cyclotron Emission and Electron Cyclotron Resonance Heating, 9 - 12 May 2006, Santorini island, Greece, p.564.

[29] Bruschi A, Cirant S, Moro A, Simonetto A, 2007, Beam Combination and Routing at High Power with a Ring-type Waveguide Millimeter-Wave Resonator, to appear in Fusion Sci. Technol. .

[30] Thumm M and Kasparek W, 2002, Passive High-Power Microwave Components. IEEE Transactions Plasma Science 30 755-786. 